\title{
Spatial correlation between malaria cases and water-bodies in Anopheles sinensis dominated areas of Huang-Huai plain, China
}

Shui-sen Zhou ${ }^{1 *}$, Shao-sen Zhang ${ }^{1+}$, Jian-jun Wang ${ }^{2}$, Xiang Zheng ${ }^{1}$, Fang Huang ${ }^{1}$, Wei-dong Li ${ }^{2}$, Xian X ${ }^{2}$ and Hong-wei Zhang ${ }^{3}$

\begin{abstract}
Background: Malaria re-emerged in the Huang-Huai Plain of central China during 2006-2008, dominated with Anopheles sinensis as a vector. However, there is no information on strategies based on multi-factor analysis to effectively control the re-emergence of malaria in these areas. Previous experience indicates some relationship between the distribution of water bodies and malaria cases, but more detailed data are not available and in-depth studies have not been conducted up to now. The objective of this study was to identify the relationship between the distribution of water bodies and presentation of malaria cases using spatial analysis tools in order to provide guidance to help formulate effective strategies for use in controlling the sources of malaria infection, based on the identification of risk areas and population.

Methods: The geographic information of malaria cases and their surrounding water bodies were collected from Suixi, Guoyang, Guzhen, Yingshang, Fengyang and Yongqiao County in Anhui province, Yongcheng and Tongbai County in Henan province. All malaria cases distributed in 113 villages in these 8 counties were collected from the China Information System for Disease Control and Prevention and confirmed by household investigation. Data on GIS and malaria cases were mapped and analyzed with the software of ArcGIS 9.2 to identify the spatial correlation between malaria cases and water bodies. The distance from households with malaria cases to the nearest water bodies was used to calculate the $O R$ value by Chi-square test. The risk area was identified through the comparison of $O R$ values in different distances.
\end{abstract}

Results: 357 malaria cases and their GPS data as well as surrounding water bodies were collected and analyzed. $74 \%$ of malaria cases were located within the extent of $60 \mathrm{~m}$ proximity to the water bodies. The risk rate of people living there and presenting with malaria was significantly higher than others $(O R=1.6,95 \% \mathrm{Cl}(1.042,2.463), P<0.05)$.

Conclusions: The results revealed that distribution of water bodies is an important factor influencing the occurrence and distribution of malaria cases in the An.sinensis areas, and implies that the scope and population within $60 \mathrm{~m}$ around water bodies are at risk and could be a targeted population for case management of malaria.

\footnotetext{
* Correspondence: ccdczss@sh163.net

${ }^{\dagger}$ Equal contributors

${ }^{1}$ National Institute of Parasitic Diseases, Chinese Center for Disease Control and Prevention; WHO Collaborating Centre for Malaria, Schistosomiasis and Filariasis; Laboratory of Parasite and Vector Biology, Ministry of Health, No.207 Rui Jin Er Road, Shanghai, People's Republic of China

Full list of author information is available at the end of the article
} 


\section{Background}

Historically the Huang-Huai Plain of central China was seriously epidemic for Malaria, and the number of cases in these areas was up to 21.99 million, accounting for $91.2 \%$ of the total reported cases in the country in 1970. With active implementation of malaria control measures for more than 30 years, considerable success had been achieved and the cases decreased dramatically and many counties have reached the standard of the basic malaria elimination (the incidence was less than 1/10000) [1]. But from early in the 21st century, malaria re-emerged in the central parts, especially the Anhui and Henan Provinces in Huang-Huai Plain located along the latitude $33^{\circ} \mathrm{N}$ with an average yearly temperature of $16{ }^{\circ} \mathrm{C}$. Total 64,178 malaria cases and 52,082 suspected cases with 38 deaths were reported in 917 counties of 23 Provinces in 2006, most malaria cases in this year with $66.4 \%$ coming from Huang-Huai Plain [2]. Afterwards, the re-emergence was effectively controlled under the enhancive control strategies and the support from the Global Funds, but the number of malaria cases and the incidence in this area has still contributed to more than $60 \%$ of the total cases in the country in recent years [3].

The main malaria vectors in this area are $A n$. sinensis and $A n$. anthropophagus in the past, but in recent years the density of $A n$. anthropophagus was very low, and the re-emergence is mainly caused by $A n$. sinensis which is developed either in the streams and pools or accumulated water on the ground [4]. These mosquitoes are less sensitive to vector controls such as indoor residual spraying (IRS) and insecticide-treated bed-nets (ITNs) because this species is campestral and its sucking habit is on cattle and pigs. Thus the control of infection sources is the major malaria control strategy in these areas $[4,5]$. Therefore, it is necessary to define the extent of infection sources for case management such as case detection, foci identification and anti-relapse treatment. The malaria cases are the major source of infection and the distribution of malaria cases matches well with the location of mosquito larval habitats [6-9] which is the leading vector in this area, inhabiting small water bodies (paddy fields, gullys, etc.) [10]. As there is limited information about the spatial characteristics of water bodies and their relationship to the distribution of malaria cases in China, the objective of this study was to explore the spatial relationship between water bodies and malaria cases.

\section{Methods}

\section{Study sites and subjects}

According to the identification of the position (upper $\backslash$ middlellower of the region) and the diversity of topographical

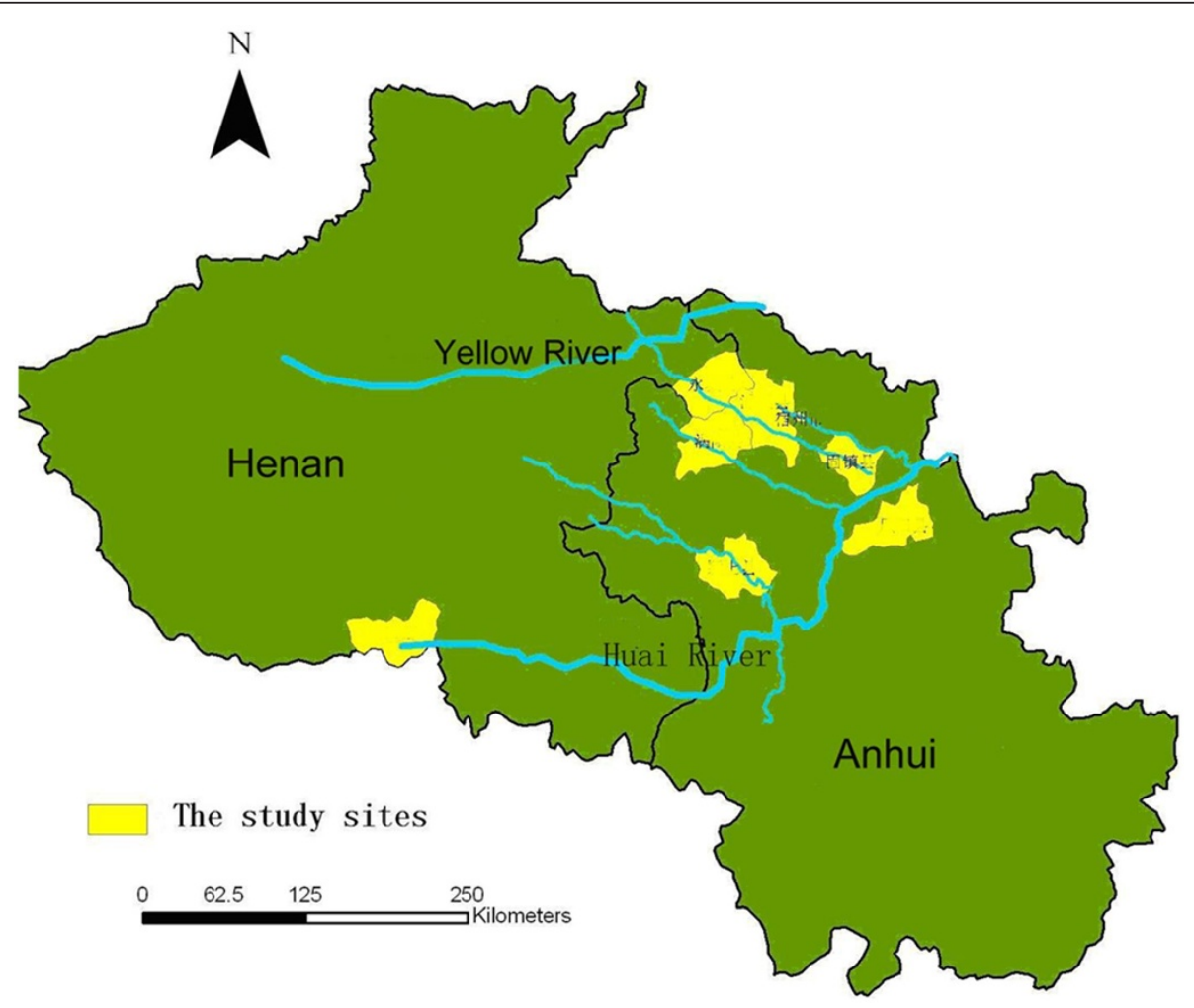

Figure 1 The map shows the location of the study sites and the Huang-Huai plain. 

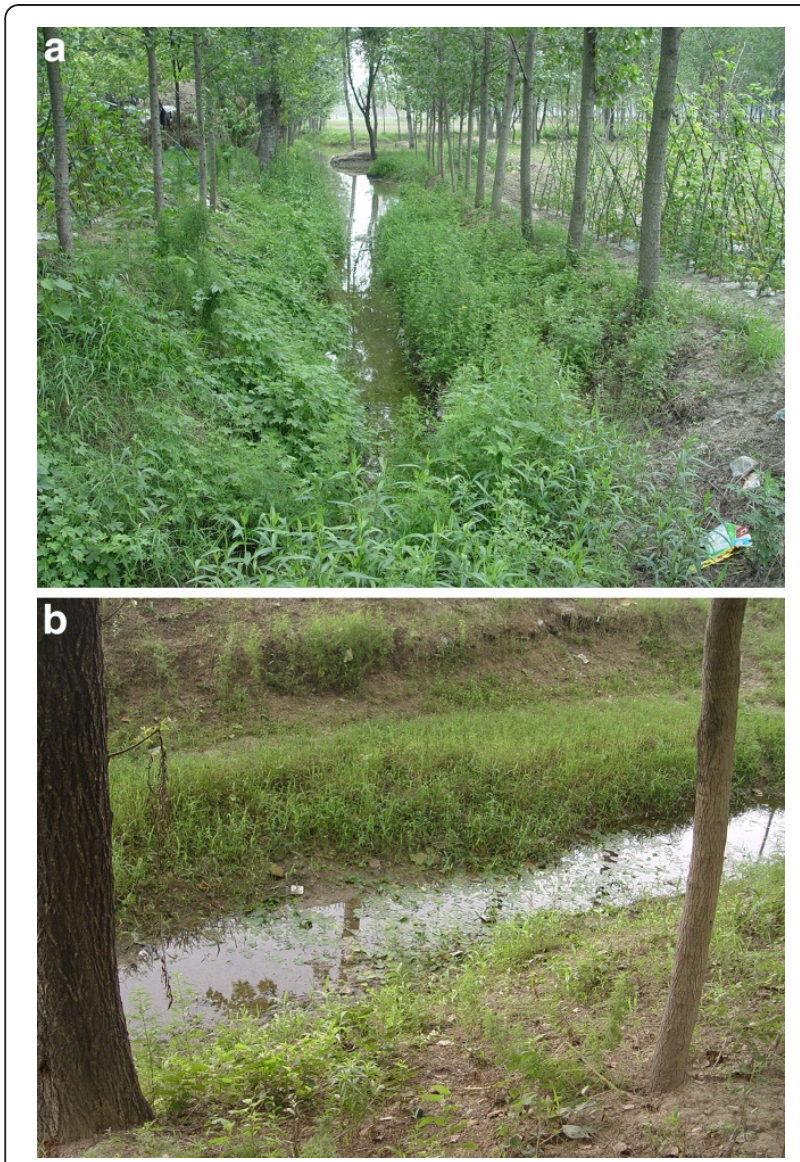

Figure 2 Water bodies. a. Shows the stream around the villages, which are considered and defined to be one kind of water body. b. Shows another kind of water body: accumulated water on the ground in the villages.

features, 113 villages (Anhui 90, Henan 23) in 8 counties (6 counties in Anhui: Fengyang, Guzhen, Suixi, Guoyang, Yingshang, Yongqiao County; 2 counties Henan: Yongcheng County and Tongbai County) were selected by stratified random unequal proportion sampling methods. All the counties were located at latitude $32^{\circ} 17^{\prime} \sim 34^{\circ} 18^{\prime} \mathrm{N}$, longitude $113^{\circ} \sim 117^{\circ} 09^{\prime} \mathrm{E}$ along the Yellow River and Huai River, with malaria re-emergence in recent years (Figure 1). The information of malaria cases in these counties were obtained from the National Malaria report system in 2004 2007, confirmed by household investigation.

\section{Data collection}

1. The data collection of water bodies

The term "water bodies" refers to the paddy fields, gully and little stream, which are suitable breeding sites of An. sinensis (Figure 2a, b). The identification of water bodies was conducted under the supervision of experienced experts who had been working on vector surveys for more than 20 years. The data was collected during the season that was suitable for malaria transmission, which is from June to October. At that time, it is easy to define the breeding sites of $A n$. sinensis by finding the larvae in the water. The data collection of malaria cases and the location of households in the village.

Based on reported malaria cases in the target villages household investigations were conducted to confirm all the epidemiological information and especially to make sure there were no invalid cases. The geographic information on all the residences including malaria cases and water bodies in the villages were collected using a hand-held GPS (Global Positioning System, GPS) locator.

\section{Data analysis}

1. Database development: The software of Mapsource was used to transform GPS data into Excel format for establishing the database.

2. Spatial analysis: The distances between houses of malaria cases and water bodies were calculated with software ArcGIS 9.2. Buffer analysis was conducted based on multiple buffer rings with water bodies to be the center. The Straight-Line Distance Function was used to calculate the distances from households of cases to the nearest water bodies, based on the results of buffer analysis.

3. Statistical analysis:

3.1 The straight-line distances from the houses of cases to the nearest water bodies were analyzed using software SPSS 11.5. The descriptive statistical analysis was conducted to get the statistical distribution of distance from house to water bodies and get the general characters of these data.

3.2 Based on the results of 3.1, all the residents in each village were grouped according to the characteristics of the distribution of malaria cases, and chi-square test was used to analyze and evaluate the risk of malaria transmission.

\section{Results}

1. The spatial correlation between malaria cases and water bodies of 246 households with malaria cases and more than 300 water bodies were collected from geographic data by GPS in the 90 villages from 6 counties in Anhui. The descriptive statistic results show that the distances from households of cases to the nearest water bodies had a positive-skew distribution (Figure 3), the median was $60.9 \mathrm{~m}$. $74.28 \%(182 / 246)$ of cases were distributed at a 

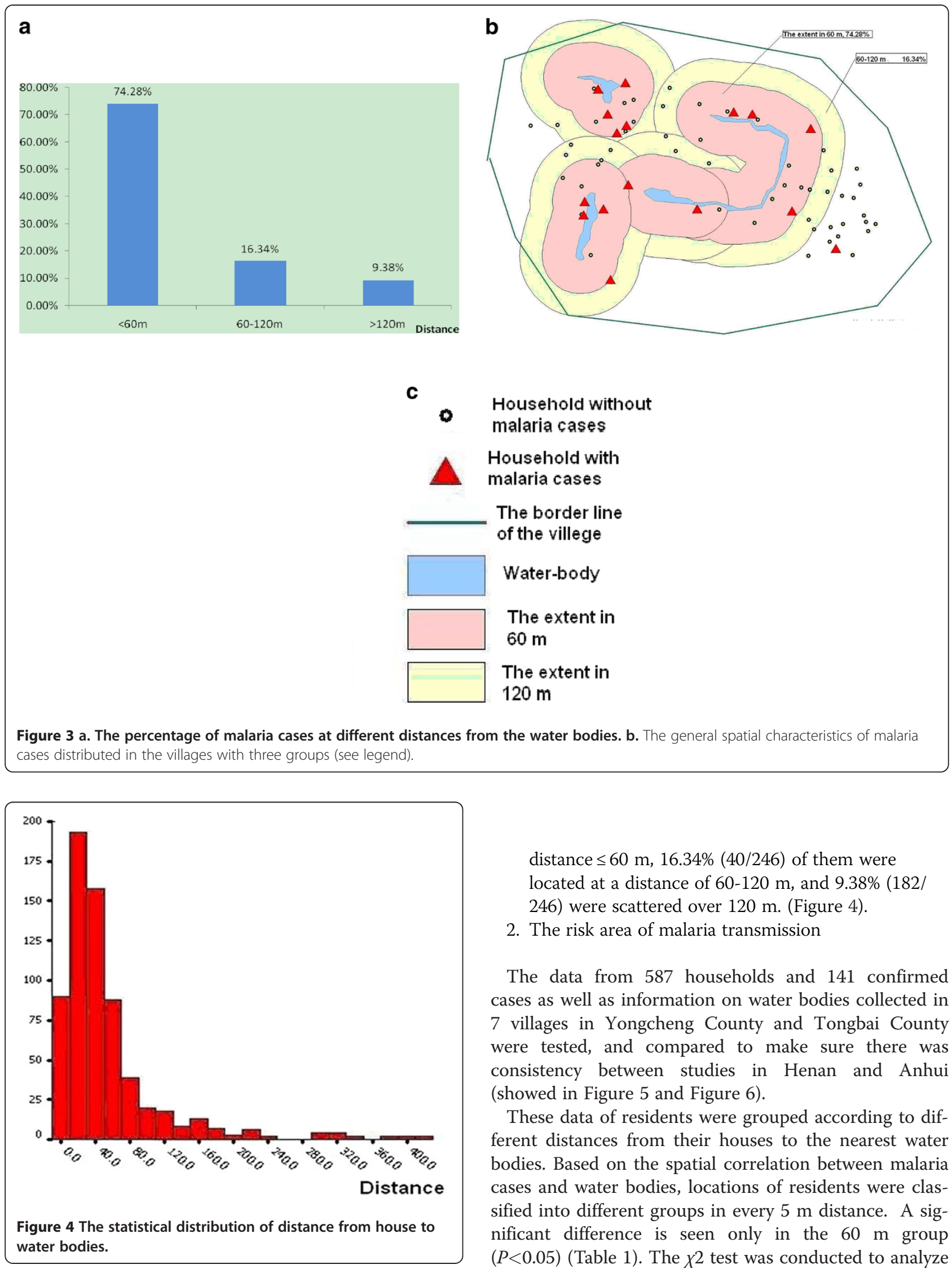

distance $\leq 60 \mathrm{~m}, 16.34 \%(40 / 246)$ of them were located at a distance of $60-120 \mathrm{~m}$, and 9.38\% (182/

246) were scattered over $120 \mathrm{~m}$. (Figure 4).

2. The risk area of malaria transmission

The data from 587 households and 141 confirmed cases as well as information on water bodies collected in 7 villages in Yongcheng County and Tongbai County were tested, and compared to make sure there was consistency between studies in Henan and Anhui (showed in Figure 5 and Figure 6).

These data of residents were grouped according to different distances from their houses to the nearest water bodies. Based on the spatial correlation between malaria cases and water bodies, locations of residents were classified into different groups in every $5 \mathrm{~m}$ distance. A significant difference is seen only in the $60 \mathrm{~m}$ group $(P<0.05)$ (Table 1$)$. The $\chi 2$ test was conducted to analyze 


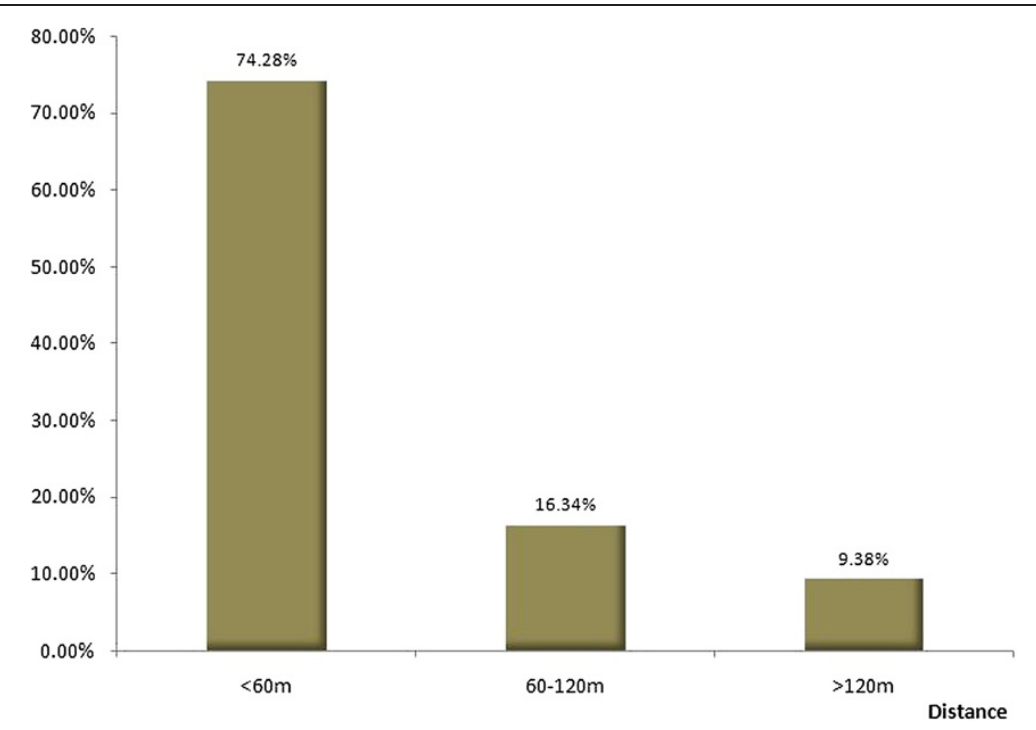

Figure 5 The percentage of malaria cases at different distances from water bodies (study sites in Anhui).

the difference between proportions of case and non-case (Table 2).

It is implied that $60 \mathrm{~m}$ may be the critical division of malaria risk areas. Grouping the residents in each village by the distance from their houses to the nearest water bodies ( $>60 \mathrm{~m}$ and $\leq 60 \mathrm{~m}$ ), the total number of households in group $\leqq 60 \mathrm{~m}$ was 391 (290 without malaria cases and 101 with malaria cases) while in the group $>60 \mathrm{~m}$, there are 196 households with 35 malaria cases. The proportion of malaria cases in the group $\leqq 60 \mathrm{~m}$ is significantly higher than that of the group $>60 \mathrm{~m}\left(\chi^{2}=4.664\right.$, $P<0.05 ; O R=1.602,95 \% \mathrm{CI}(1.042,2.463), P<0.05)$. It concluded that people living within the extent of $60 \mathrm{~m}$ near water bodies have more risk of malaria infection.

\section{Discussion}

As a vector-borne disease, malaria transmission has a close relationship with the biological and natural factors due to the vectors-anopheles. Currently An. sinensis is the sole or major vector in the Huang-Huai Plain, this mosquito is campestral and its blood feeding habit is on cattle and pigs, and its larval habitats are the small water bodies such as ponds, paddy fields, gullies, and so on $[11,12]$. Vector control measures such as ITN are not

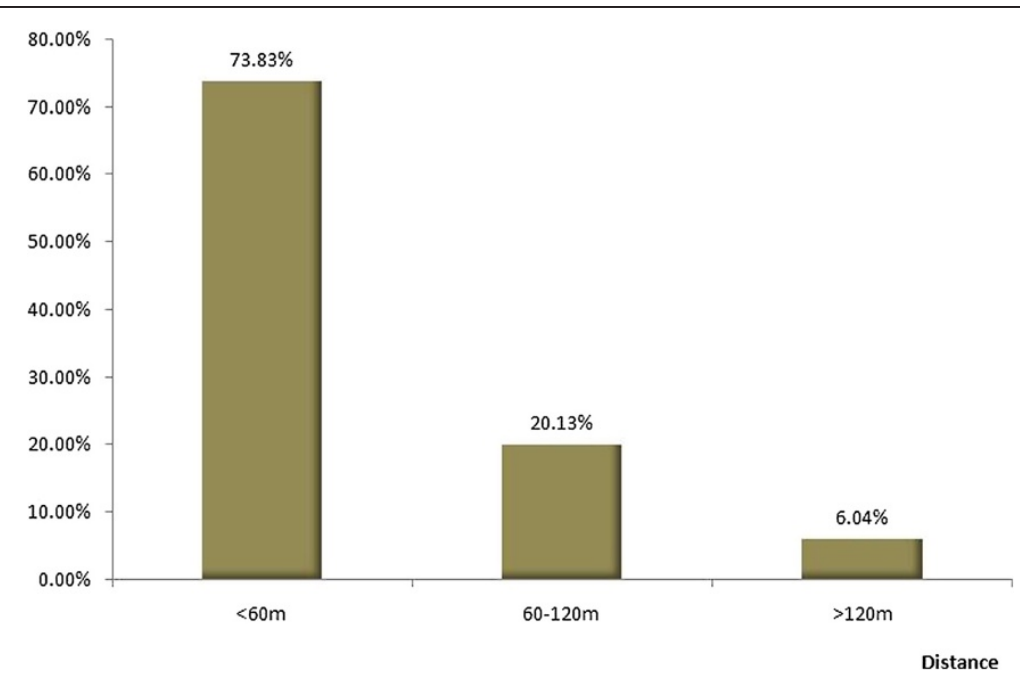

Figure 6 The percentage of malaria cases at different distances from water bodies (study sites in Henan). 
Table 1 The Chi-square and $O R$ value of Different group division

\begin{tabular}{lccccc}
\hline Group & chi-square & $\boldsymbol{P}$ value & OR & \multicolumn{2}{c}{$\mathbf{9 5 \% C l}$} \\
\hline $50 \mathrm{~m}$ & 0.919 & 0.338 & 1.16 & 0.851 & 1.593 \\
\hline $55 \mathrm{~m}$ & 3.125 & 0.077 & 1.332 & 0.964 & 1.84 \\
\hline $60 \mathrm{~m}$ & 4.664 & 0.041 & 1.602 & 1.042 & 2.463 \\
\hline $65 \mathrm{~m}$ & 3.398 & 2.306 & 2.706 & 1.869 & 3.918 \\
\hline
\end{tabular}

The Table 1 showed Chi-square and OR value of Different group division and presented that how the cut-off $60 \mathrm{~m}$ was identified.

Table 2 Comparison of the risk between two groups

\begin{tabular}{cccc}
\hline Group & \multicolumn{2}{c}{ No. of households } & Total \\
\cline { 2 - 3 } & No-cases & Cases & \\
\hline$\leqq 60 \mathrm{~m}$ & 290 & 101 & 391 \\
\hline$>60 \mathrm{~m}$ & 161 & 35 & 196 \\
\hline Total & 451 & 136 & 587 \\
\hline
\end{tabular}

The Table 2 showed the $x 2$ analysis of two groups (no-cases and cases).

effective in these areas. Thus case management is critically important especially in the interventions to risk population around mosquito breeding -water bodies. The previous studies $[9,13,14]$ found a negative relationship between the risk of malaria infection and the distance from the anopheles breeding sites to the houses: the closer residents live to anopheles breeding sites, the higher the risk of malaria infection becomes, due to the higher density of anopheles. Entomological survey found that the anopheles prefer to lay eggs near the blood meal sites, which results in an increase of vector density, thus causing the increase of malaria risk among people living close to larval habitats. However, little information about the relationship between larval habitat distribution and malaria cases can be obtained in this area in the historical literature. We evaluated the relationship between malaria prevalence and the distribution of water bodies, and obtained similar results to the previous studies on other anopheles spp. [15-21].

All of the study sites belong to under-developed counties and have similar social factors, such as the cultivation (the main crops are wheat, rice, corn, soybean, etc.), architectural style of houses (only windows in the front of house), and daily habits (most of the residents prefer to sleep outside in summer). So, excluding the effect of social factors, the results of our study indicated that the increase of malaria risk near the water bodies could be attributed to the anopheles habitat.

The Huang-Huai Plain has been malaria endemic many times, according to the previous national reports. The main strategies used for control was anti-malaria treatment, given to all the people or administration of the medicine to all the people for prevention [10]. However, as these strategies were expensive and had low compliance, they were abandoned in some counties.
Some suggestions based on our results have been given for rolling back re-emergence and controlling transmission of malaria, this includes: the high risk population should be identified based on the distance from housing to water bodies $(\leq 60 \mathrm{~m})$ which is more cost effective; People living in the risk area are the targeted population who should take some preventative treatment; mosquito breeding sites around housing needs to be cleared, taking measures particularly against larvae.

\section{Conclusion}

The Huang-Huai Plain was malaria endemic area, according to the previous national reports. The main strategies used for control was anti-malaria treatment, given to all the people or administration of the medicine to all the people for prevention [10]. This study combined investigation in the field and spatial analysis on computer, to describe the character of malaria cases distribution in the village level and develop the risk map. The results revealed that distribution of water bodies is an important factor influencing the occurrence and distribution of malaria cases in the An.sinensis areas, and implies that the scope and population within $60 \mathrm{~m}$ around water bodies are at risk and could be a targeted population for case management of malaria.

\section{Competing interests}

The authors declare that they have no competing interests.

\section{Acknowledgements}

We acknowledged the contribution of Xian Xu, Wei-dong Li and Zhou Guang-chao for the field work support. The smooth collaboration with the staff of the Henan province and Anhui province CDC is acknowledged. This work was supported by the Special Social Commonweal Research Programs in Ministry of Science and Technology of P.R.China (No.2005 DIB1J092), the National S \& T Major Program (No. 2008ZX10004-011) and Operational Research Program supported by China Global Fund Malaria Program (No. 20120302).

\section{Author details}

${ }^{1}$ National Institute of Parasitic Diseases, Chinese Center for Disease Control and Prevention; WHO Collaborating Centre for Malaria, Schistosomiasis and Filariasis; Laboratory of Parasite and Vector Biology, Ministry of Health, No.207 Rui Jin Er Road, Shanghai, People's Republic of China. ${ }^{2}$ Anhui Province Center for Disease Control and Prevention, No.12560 Fanhua Road in EconomicTechnological Development Zone, Hefei, People's Republic of China. ${ }^{3}$ Henan Province Center for Disease Control and Prevention, Eastern Nongye Road, Zhengzhou, People's Republic of China.

\section{Authors' contributions}

SSZ was responsible for the overall study and involved in all stages of this study including design, field work, data analysis and writing the manuscript. SSZ was the focal point in this study and involved in field work and data analysis and wrote the manuscript. JJW, XZ, FH, WDW, XX and HWZ performed the collection of malaria incidence data in study sites. All authors read and approved the final manuscript.

Received: 3 November 2011 Accepted: 31 May 2012 Published: 31 May 2012

\section{References}

1. Tang LH: Progress in malaria control in China. Chin Med J 2000, 115:69-92. 
2. Zhou SS, Wang Y, Tang LH: Malaria situation in the People's Republic of China in 2006. Chin J Parasitol and Parasit Dis 2007, 25(6):439-411.

3. Zhou SS, Wang Y, Xia ZG: Malaria situation in the People's Republic of China in 2009. Chin J Parasitol and Parasit Dis 2011, 29(1):1-3.

4. Ministry of Health Disease Prevention and Control Bureau: Handbook for malaria control and prevention. Beijing: People's Hygine Publishing House; 2007:98-99.

5. Pan B: The morphological characteristics, ecological habit, malaria transmission effect of malaria vectors in China. J Trop Med 2003, 3(4):477-480.

6. Allen WH, Maurice O, Richard O, Richard O, Aggrey JO, Altaf AL, Bernard LN, William AH: A Geographic Information System applied to a malaria field study in western Kenya. Am J Trop Med Hyg 1998, 58(3):266-272.

7. Foley D, Torres E, Mueller I, Bryan JH, Bell D: Host-dependent Anopheles flavirostris larval distribution reinforces the risk of malaria near water. Trans Roy Soc Trop Med Hyg 2003, 97:283-287.

8. Noboru M, Pamela S, Guiyun Y: Influence of host and larval habitat distribution on the abundance of African malaria vectors in western Kenya. AmJTrop Med Hyg 2002, 67(1):32-38.

9. Thomas C, Lindsay S: Local-scale variation in malaria infection amongst rural Gambian children estimated by satellite remote sensing. Trans $R$ Soc Trop Med Hyg 2000, 94:159-163.

10. Zhou ZJ: Study of malaria control and prevention in China. Beijing: People's Hygine Publishing House; 1991:98-117. ISBN 7-117-01501-2.

11. Gou GX, Li DF, Shang LY, Guo XS, Wang WX, Sun QL: The study on ecological habits of Anopheles sinensis in Guantang, Luyi County from 1971 to 1996. Chin J Vector Biol \& Control 1998, 9(2):133-134.

12. Hu YX, Miao YG, Fan TB: The further study on ecological habits of Anopheles sinensis in the area along Yellow River and Huai River. Chin $J$ Parasitol Parasit Dis 1988, S1:135.

13. Foley $\mathrm{D}$, Torres $E$, Mueller I: Stream-bank shade and larval distribution of the Philippine malaria vector Anopheles flavirostris. Med Vet Entomol 2002, 16:347-335.

14. Jean-Francois T, Lefebvre-zante E, Fabrice L, Gora N, Hilaire B, Pierre D, Gerard S: Vector density gradients and the epidemiology of urban malaria in Dakar, Senegal. AmJTrop Med Hyg 1992, 47(2):181-189.

15. Noboru M, Clifford M, John I, John CB, Guiyun Y: Spatial distribution and habitat characterization of Anopheline mosquito larvae in western Kenya. Am J Trop Med Hyg 1999, 61(6):1010-1016.

16. Richard C, Kamini N, Donald R: Spatial targeting of interventions against malaria. Bull World Health Org 2000, 78(12):1401-1411.

17. Gunawardena D, Wickremasinghe A, Muthuwatta L, Weerasingha S, Rajakaruna J, Senanayaka T, Kotta PK, Attanayake N, Carter R, Mendis KN: Malaria risk factors in an endemic region of Sri Lanka, and the impact and cost implications of risk factor-based interventions. Am J Trop Med Hyg 1998, 58(5):533-542.

18. Michael TW, Jamie TG, Thomas SC, Neil MF, Maria-Gloria B, Azra CG: Modelling the impact of vector control interventions on Anopheles gambiae population dynamics. Parasit Vectors 2011, 4:153.

19. Eliningaya JK, Zhou GF, Thomas MG, Yaw A, Mramba N, Andrew KG, Guiyun $Y$ : Predation efficiency of Anopheles gambiae larvae by aquatic predators in western Kenya highlands. Parasit Vectors 2011, 4:128.

20. Louis CG, Jean-Sébastien D, Romain G, Sebastien B, Guy L, Didier F: Spatial and temporal distribution patterns of Anopheles arabiensis breeding sites in La Reunion Island - multi-year trend analysis of historical records from 1996-2009. Parasit Vectors 2011, 4:121.

21. Marianne ES, Michael JB, Sylvie M, Theeraphap C, Anand PP, William HT, Peter WG, Iqbal RFE, Caroline WK, Ralph EH, Simon IH: The dominant Anopheles vectors of human malaria in the Asia-Pacific region: occurrence data, distribution maps and bionomic précis. Parasit Vectors 2011, 4:89.

doi:10.1186/1756-3305-5-106

Cite this article as: Zhou et al:: Spatial correlation between malaria cases and water-bodies in Anopheles sinensis dominated areas of Huang-Huai plain, China. Parasites \& Vectors 2012 5:106.

\section{Submit your next manuscript to BioMed Central and take full advantage of:}

- Convenient online submission

- Thorough peer review

- No space constraints or color figure charges

- Immediate publication on acceptance

- Inclusion in PubMed, CAS, Scopus and Google Scholar

- Research which is freely available for redistribution

Submit your manuscript at www.biomedcentral.com/submit
C Biomed Central 